\title{
Can intrinsic foraging efficiency explain dominance status? A test with functional
}

response experiments

Alexandra Hartley ${ }^{1}$, Adrian M. Shrader ${ }^{1,2}$, Simon Chamaillé-Jammes ${ }^{2,3 *}$

${ }^{1}$ School of Life Sciences, University of KwaZulu-Natal, Private Bag X01, Scotsville 3209, South Africa

${ }^{2}$ Mammal Research Institute, Department of Zoology \& Entomology, University of Pretoria, Private Bag X28, Pretoria, 0028, South Africa

${ }^{3}$ CEFE, CNRS, Univ Montpellier, Univ Paul Valéry Montpellier 3, EPHE, IRD, Montpellier, France

*Corresponding author: Simon Chamaillé-Jammes; Address: Centre d'Ecologie Fonctionnelle et Evolutive, 1919 Route de Mende, 34293 Montpellier Cedex 5, France.

e-mail: simon.chamaille@ @efe.cnrs.fr; phone: (+33)(0)467613218

Author Contributions: AMS and SCJ designed the study. AH performed the experiments. SCJ analysed the data and wrote the initial draft of the manuscript. All authors contributed to the final version of the manuscript. 


\begin{abstract}
The functional response describes how food abundance affects the intake rate of foraging individuals, and as such, it can influences a wide range of ecological processes. In social species, dominance status can affect the functional response of competing individuals, but studies conducted in an interferencefree context have provided contrasting results on the extent of between-individual variability in functional response. We tested the prediction that individuals intrinsically differ in their functional response, and that these differences could predict body weight and dominance status in social species. We used goats as a model species and performed foraging experiments to assess the functional response of these goats in an interference-free context. Our results show that some individuals are consistently better foragers than others, and these individuals were more likely to be heavier and dominant. Parameters of the functional response are however more strongly associated with dominance status than with body weight. We conclude that interference while foraging is not needed to explain body weight differences between dominant and subordinate individuals. We suggest that these differences can emerge from intrinsic differences in foraging efficiency between individuals, which could also allow better foragers to demonstrate greater tenacity during agonistic interactions.
\end{abstract}

Key words: competition; goat; interference; inter-individual variability; hierarchy 


\section{Introduction}

The functional response describes how food abundance affects the intake rate of foraging individuals (Stephens \& Krebs 1986). As such, the functional response has a critical influence on a wide range of ecological processes, including distribution and movement of individuals within landscapes (Calcagno et al. 2014), predator-prey dynamics (Abrams 2000), and the stability of complex food webs (Post et al. 2000, Brose et al. 2003).

Many theoretical and empirical studies assume that functional responses are species or class (e.g. ontogenic stages, ages, sexes) specific, commonly accounting only for large body size differences, as these are known to be important (Kalinkat et al. 2013). However, little is known about interindividual variability of functional response between individuals of similar body size. Studies have shown that dominance status affects the foraging efficiency of interfering individuals (e.g. Vahl et al. 2005, Nilsson et al. 2004), but very few studies have investigated the existence of intrinsic interindividual variability in the absence of interference. Those that have, however, obtained contrasting results (e.g. differences between individuals found in Ranta and Nuutinen 1985, Fritz et al. 2001, Arzel et al. 2007, Schröder et al. 2016; no differences found in Nilsson et al. 2004). This leaves an important gap in our understanding of foraging and its ecological consequences. For instance, intrinsic differences in foraging efficiency could help explain inter-individual differences in weight gain, which is often associated with better demographic performance (Saether 1989, Pelletier et al. 2007). In gregarious species it could also influence dominance status, as heavier individuals tend to be dominant (Veiberg et al. 2004, Vervaecke at al. 2005, Favre et al. 2008). However, individuals may not be consistently better or worse off than others at foraging across a gradient of resource densities. In this case, characterized by the crossing of individual functional response curves, predicting distribution of individuals in heterogeneous patches, and the outcome of exploitation and interference competition between individuals, would be more complex. 
Here we tested the prediction that individuals intrinsically differ in their functional

response, and that these differences predict body weight and dominance status in social species. We did this by using goats as a model system and estimating two key functional response parameters at the individual level, attack rate (instantaneous rate of food discovery) and handling time (time required to bite and ingest food). Goats were isolated during these experiments, in order to provide an interferencefree context, and thus directly measure their intrinsic foraging efficiency.

\section{Materials and methods}

\section{Foraging assays}

We conducted foraging assays using 18 adult female goats, housed at the Ukulinga experimental farm (University of Kwazulu-Natal, South Africa). We weighed the goats at the start of the study, early morning before they fed to avoid biases due to rumen fill. In order to build functional response curves, we estimated the intake rate of each goat at increasing food abundance. To do this, we placed 50, 100, 200, 400 or $800 \mathrm{~g}$ of sheep pellets (Complete Sheep Finisher, Meadow Feeds, South Africa) in feeding trays $(570$ x 365 x $230 \mathrm{~mm})$ similar to those used by Shrader et al. (2012) and Stears et al. (2014). To provide diminishing returns and induce a type-II functional response across the range of food abundance, we attached a $3 \times 3 \mathrm{~cm}$ grid of $2.5 \mathrm{~mm}$ galvanized wire over the top of each tray and added 5 L of inedible medium (i.e. $20 \mathrm{~cm}$ lengths of $30 \mathrm{~mm}$ plastic tubing) inside the tray. For three weeks prior to the trials, goats were trained to familiarize with the structure of the pen (i.e. $4.5 \times 7 \mathrm{~m}$ with the sides covered by black shade cloth), the feeding trays, and the presence of humans while feeding.

For the first week, groups of three or four individuals were allowed into the pen to feed from the same feeding tray. The next week, we ushered goats one by one into the feeding area and allowed them to feed for a maximum of one minute. From the third week onwards, when we collected data, we limited the effect of food depletion by allowing individuals to feed for only 30 seconds before we 
removed the tray. We then weighed the amount of food left in the tray and calculated the intake rate $(\mathrm{g} / \mathrm{sec})$ of the goat during the trial. For each goat and each food abundance, the assay was replicated 5 times.

On any given day, all 18 goats were tested, but each goat was only tested once. This prevented the intake of previous trials influencing any subsequent trials. To prevent the goats from being able to predict the different food abundances, the amount of food presented to each goat each day was randomized. All experiments were conducted between 07:00 and 11:00. To ensure that the goats were hungry during this period, they were housed overnight in a $30 \times 12 \mathrm{~m}$ sheltered barn where they had ad libitum access to water, but no food. This is their normal routine and mimics common husbandry practices (see Shrader et al. 2008). In the mornings prior to the experiments, individuals were moved into a rye grass pasture where they fed for 30 minutes. This allowed them to eat some food, but not fill their stomachs. Ultimately, this ensured that all goats tested were hungry but that goats tested at the end of the morning sessions did not reach very high hunger levels. Additionally, in a given day, goats were tested in a random order. After the experiments, the goats were then released into natural veld, enabling them to feed undisturbed.

The dominance hierarchy of the goat herd used here had been determined by Stears et al. (2014). However, to ensure that the hierarchy was the same during our study, we reconfirmed the relative ranks of the individuals used in our experiments. As with Stears et al. (2014), we did this by facilitating interactions between two goats at a single artificial food patch. In one patch, we poured 200 $\mathrm{g}$ of sheep pellets into one of the corners, and released two goats from opposite sides of the patch. Dominance was then determined by observing the interactions between these two individuals (see Stears et al. 2014 for details). Dominant individuals were classified as those individuals that initiated aggressive acts (e.g. horn butting, charges), and/or prevented the other individual from feeding from the patch. Subordinate individuals either did not approach the patch, or were chased away from the patch 
by the dominant individual.

Data are available on figshare.com, with doi 10.6084/m9.figshare.6795056.

\section{Statistical analyses}

We fitted a type II functional response (Holling 1959) to the data, using the classical formulation:

$$
\mathrm{I} \sim(\mathrm{a} * \mathrm{~F}) /\left(1+\mathrm{a}^{*} \mathrm{~h} * \mathrm{~F}\right)
$$

with I the intake rate $\left(\mathrm{g} . \mathrm{s}^{-1}\right)$, a the attack rate $\left(\mathrm{s}^{-1}\right)$, h the handling time (s), and $\mathrm{F}$ the food abundance (g). We fitted this model in a non-linear mixed model framework, allowing for random effects on handling time and attack rate per individual. The increase in the intake rate variance as food abundance increased was accounted for by using a variance identity function (Zuur et al. 2009). We tested the significance of inter-individual variability in attack rate and handling time, and of the correlation between attack rates and handling times (measured using Pearson's r), using permutation tests. At each food abundance, the identity of the goats was randomly permuted, the model re-run, and attack rates, handling time, and the correlation between them estimated. This was done 5000 times in order to obtain the distribution of the random effects variance (for both attack rate and handling time) and of the correlation under the null hypothesis of no inter-individual variability in the functional response. The variances of the individual random effects from the model fitted to the real data, and the correlation between attack rates and handling times, were then compared to the distributions built under the null hypothesis and approximate P-values were calculated.

Finally, we investigated if random effects on attack rates and handling times were best explained by dominance status or body weight. For attack rate and handling times, we built linear models with either dominance or body weight as unique predictor of the random effect values, and compared both models using the Akaike Information Criterion. Statistical analyses were conducted using the R software (R Core Team 2017). 


\section{Results}

Permutation tests revealed that there was significant inter-individual variation in attack rates $(\mathrm{P}<$ $0.001)$ and handling times $(\mathrm{P}=0.030)$, which were negatively correlated at the individual level $(\mathrm{r}=-$ $0.82, \mathrm{P}=0.057$; Fig. 1 inset). Hence, some individuals were consistently foraging more rapidly than others at all food densities, with the foraging rate of the most efficient goat being approximately 3 times higher than the least efficient one at the highest food density (Fig. 1). Dominant goats tended to be heavier than subordinate ones (ANOVA; $\mathrm{F}_{1,16}=3.827, \mathrm{P}=0.068$ ), but attack rates and handling times were best related to dominance status rather than to weight ( $\triangle \mathrm{AIC}$ between models with either dominance or weight as unique predictor were 5.5 and 4.5 for attack rates and handling times respectively). Dominant goats had higher attack rates and shorter handling times than subordinates (ANOVAs; $\mathrm{F}_{1,16}=12.400, \mathrm{P}=0.003$ and $\mathrm{F}_{1,16}=15.890, \mathrm{P}=0.001$ respectively; Fig. 2).

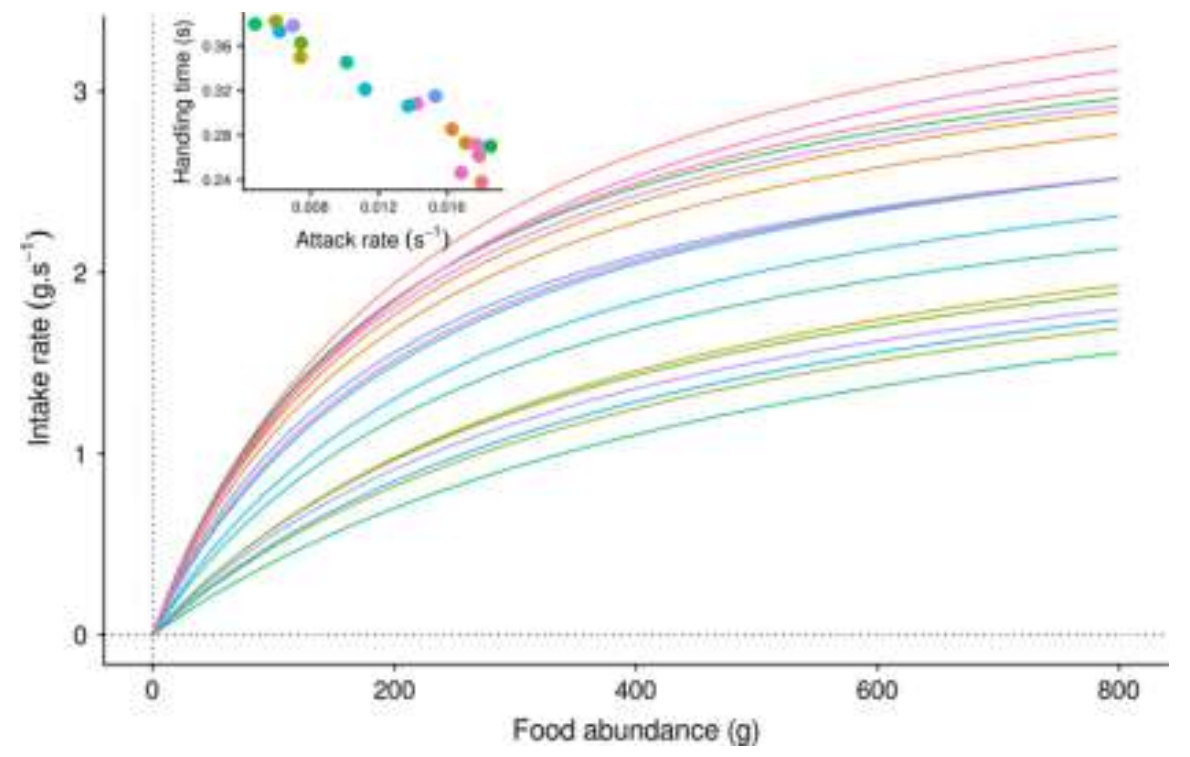

Figure 1. Functional response curves of 18 goats, built from foraging experiments replicated five times for each individual at varying food abundance (at 50, 100, 200, 400 and $800 \mathrm{~g}$ ). Curves followed the classical type-II formulation: I (a*F) / $\left(1+a^{*} h * F\right)$, with I the intake rate $\left(\right.$ g.s $\left.{ }^{1}\right)$, a the attack rate $\left(\mathrm{s}^{-1}\right), \mathrm{h}$ the handling time (s), and F the food abundance $(\mathrm{g})$. Attack rate and handling times were estimated using a non-linear mixed model approach, with random effects on both allowed for each individual. Inset: Relationship between attack rate and handling time. The same colour identify the same individual in the main figure and in the inset. 

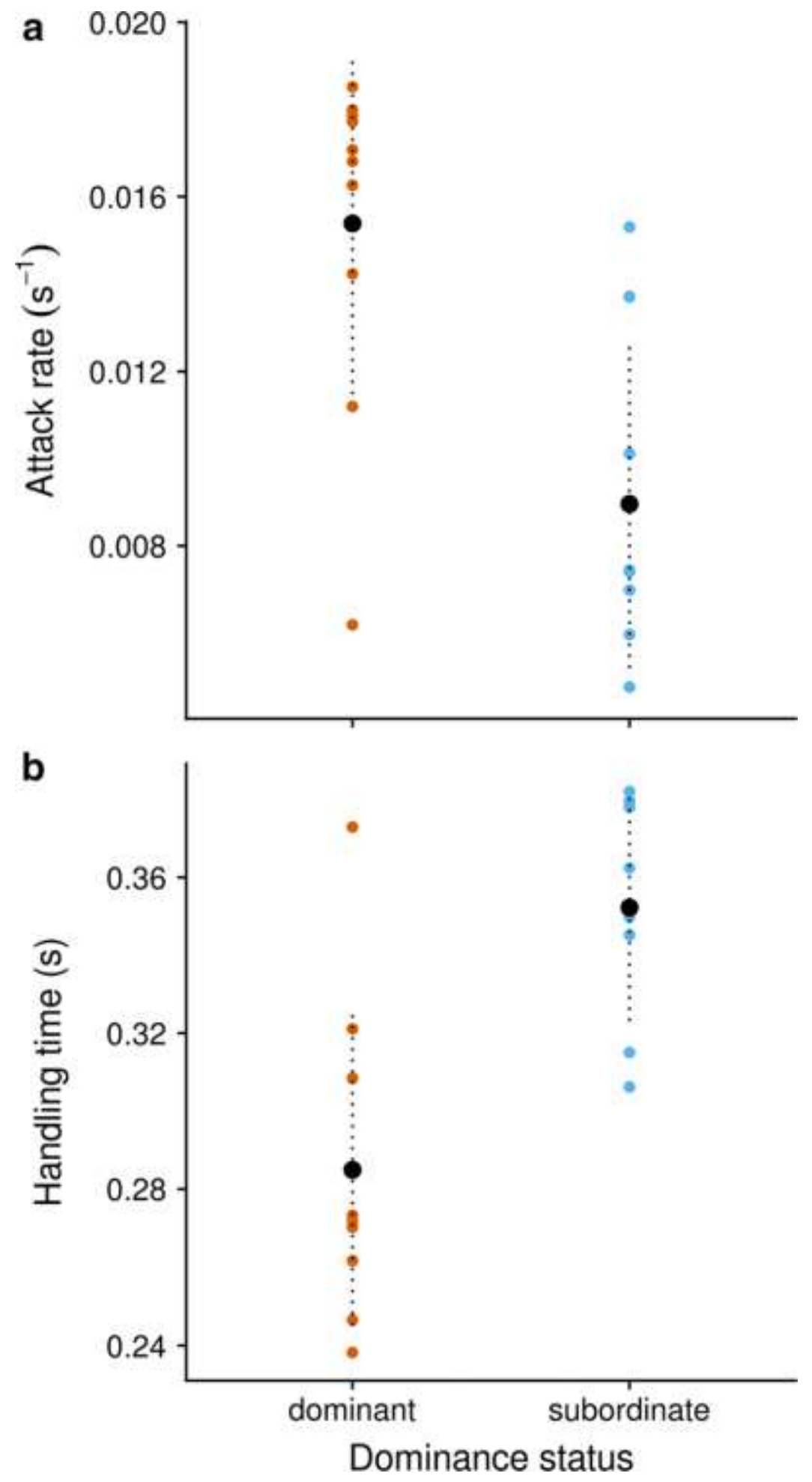

Figure 2. Differences in (a) attack rates and (b) handling times between dominant and subordinate individuals. Individual data (coloured dots) as well as mean \pm S.D. (black dot and dotted line) are shown. 


\section{Discussion}

The functional response is a key pillar of the ecological theory (Stephens \& Krebs 1986, Abrams 2000). Many studies assume that it is species or sex/age specific, but our results demonstrate that there could also be significant inter-individual differences, with some individuals consistently foraging more efficiently than others. The mechanistic basis of this difference is that individuals with shorter handling times also have higher attack rates. Individual differences between individual functional responses had been found by a few other studies (Ranta and Nuutinen 1985, Fritz et al. 2001, Arzel et al. 2007, Schröder et al. 2016), but others failed to find differences (Nilsson et al. 2004). The current paucity of studies on this issue however, prevent assessing the commonness of inter-individual differences, and call for further studies. We however suggest that the mixed-model approach used here or in Schröder et al. (2016), now widely recognized as the state-of-the-art method to study individual heterogeneity (Dingemanse \& Dochtermann 2013), should be favoured over previous approaches which suffered from a lack of replication at the individual level (Ranta and Nuutinen 1985, Fritz et al. 2001), or from an averaging of the individual data (Arzel et al. 2007, Nilsson et al. 2004).

Our results suggest that dominant individuals, who are generally heavier, are intrinsically better foragers than subordinates (i.e. there are between-individual intrinsic differences), at all food densities considered. During the trials, goats foraged alone and thus antagonistic behaviours between individuals, or body weight differences that may determine winners in these aggressive interactions between individuals, did not affect our functional response measures. Thus, our findings contrast with those of Stillman et al. (1996), where dominance status did not affect the foraging efficiency of oystercatchers in interference-free context. Moreover, in contrast to Rands et al. (2006), our study indicates that interference is not needed to explain body weight differences between dominant and subordinate individuals. Nevertheless, it is likely that interference between individuals would increase differences over time. Thus, one is led to assume that the link between the functional response of a 
foraging individual and its dominance status occurs through the faster and possibly greater increase in body weight of efficient foragers, which then dominate others during antagonistic interactions.

However, we show here that parameters of the functional response are more strongly related to dominance than to weight, suggesting that other processes may also play a role. It is possible that more efficient foragers, gaining more food per unit of foraging time than other individuals, are more willing to sacrifice foraging time to engage in agonistic interactions. As tenacity during interactions is likely to be critical in determining winners and losers (Francis 1988), this could explain the relationship between foraging efficiency and dominance observed here.

It is also possible that dominance results from the expression of 'personality' traits, such as aggressiveness, that could be associated with foraging efficiency in a behavioural syndrome (Sih et al. 2004). Mechanistically, such correlation between foraging, behaviour, and ultimately dominance could emerge from differences in the pace-of-life of individuals (Dammhahn et al. 2018). Individuals of a higher pace-of-life usually forage more or faster, and have indeed sometimes been shown to be 'bolder', more aggressive, and to more easily achieve dominant status (Metcalfe et al. 2016). This is however, not always observed (Royauté et al. 2018), and further studies will be required to understand the processes underlying the patterns observed here.

Although we have considered that foraging efficiency was an intrinsic characteristic of the individual that could explain body size and dominance status, we should also consider the alternative explanation that increased foraging efficiency emerged from, rather than drove, increasing body size. It is however, unclear what factor could link body size to functional response. It has been suggested that anatomical differences linked to body size, such as bill size in birds (Durant et al. 2003), or incisors arcade width in ungulates (Gordon et al. 1996) could determine foraging efficiency. We measured the incisor arcade of 9 of our goats (the others had been sold prior to us being able to collect these data), but found no relationship between the parameters of the functional response and the incisor arcade 
width of the goats (linear regressions; attack rates: $\mathrm{F}_{1,8}=0.1679, \mathrm{P}=0.693$; handling times: $\mathrm{F}_{1,8}=1.026$, $\mathrm{P}=0.341$ ). Therefore, we favour the hypothesis that foraging efficiency contributes to determine body weight and dominance status.

Although we have clearly demonstrated consistent inter-individual differences in foraging efficiency, this conclusion remains limited to a unique, rather simple, foraging situation. In the wild, individuals are faced with a heterogeneous environment in which they exploit many food types, and extrapolating our results to performance of individuals in complex environments is premature. For instance, Fritz et al. (2001) suggested that inter-individual differences in foraging efficiency of mallards might vary with food item size. However, in their set of item size, individual efficiency sometimes decreased, but the ranking of individuals did not change across item sizes. Thus, further studies investigating whether poorer foragers on one food item can be better foragers on others remain to be conducted.

We conclude by emphasizing, following Kalinkat (2014), the value of studying the variability of the functional response between individuals. This approach has the potential to shed new light on the links between foraging and other behavioural characteristics of individuals. As shown here, this approach can challenge our understanding of extensively studied topics, such as the relationship between foraging and dominance, but can also contribute to more recent fields of research (e.g. foraging-personality: Toscano and Griffen 2014). Moreover, implications for higher-order processes should be understood. For instance, it can be envisioned that inter-individual differences in functional responses will affect predator-prey relationships, as better foragers should have more time to devote to vigilance, and be able to use richer-but-riskier patches more. The distribution of functional response 'phenotypes', irrespectively of 'personalities', in the prey population could then be a strong determinant of prey space use, predation rates and predator-prey dynamics, but this remains to be tested. Generally, a great challenge lies in understanding to what extent, and under what conditions, 
will such individual differences in functional responses matter for population and ecosystem processes.

\section{Acknowledgements}

We thank K. Stears for helping with the dominance hierarchy of the goat herd. This research was funded by the CNRS 'Groupe de Recherche International France - Afrique du Sud' (SCJ), and the National Research Foundation (Grant 77582: AMS). Comments from A. Nilsson and an anonymous reviewer improved the manuscript.

\section{References}

Abrams PA (2000) The evolution of predator-prey interactions: Theory and Evidence. Annual Review of Ecology, Evolution, and Systematics 31:79-105.

Arzel C, Guillemain M, Gurd DB, Elmberg J, Fritz H, Arnaud A, Pin C, Bosca F (2007) Experimental functional response and inter-individual variation in foraging rate of teal (Anas crecca). Behavioural Processes 75:66-71. https://doi.org/10.1016/j.beproc.2007.01.001

Calcagno V, Grognard F, Hamelin FM, Wajnberg É, Mailleret L (2014) The functional response predicts the effect of resource distribution on the optimal movement rate of consumers. Ecology Letters 17:1570-1579. https://doi.org/10.1111/ele.12379

Dammhahn M, Dingemanse NJ, Niemelä PT, Réale D (2018) Pace-of-life syndromes: a framework for the adaptive integration of behaviour, physiology and life history. Behavioral Ecology and Sociobiology 72:62. https://doi.org/10.1007/s00265-018-2473-y

Dingemanse NJ, Dochtermann NA (2013) Quantifying individual variation in behaviour: mixed-effect modelling approaches. Journal of Animal Ecology 82:39-54. https://doi.org/10.1111/1365-2656.12013 Durant D, Fritz H, Blais S, Duncan P (2003) The functional response in three species of herbivorous Anatidae: Effects of sward height, body mass and bill size. Journal of Animal Ecology 72:220-231. 
https://doi.org/10.1046/j.1365-2656.2003.00689.x

Francis RC (1988) On the relationship between aggression and social dominance. Ethology 78:223237.

Fritz H, Durant D, Guillemain M (2001) Shape and sources of variations of the functional response of wildfowl: an experiment with mallards, Anas platyrhynchos. Oikos 93:488-496. https://doi.org/10.1034/j.1600-0706.2001.930314.x

Gordon IJ, Illius AW, Milne JD (1996) Sources of variation in the foraging efficiency of grazing ruminants. Functional Ecology 10:219-226.

Kalinkat G (2014) Bringing animal personality research into the food web arena. Journal of Animal Ecology 83:1245-1247.

Metcalfe NB, Van Leeuwen TE, Killen SS (2016) Does individual variation in metabolic phenotype predict fish behaviour and performance? Journal of Fish Biology 88:298-321. https://doi.org/10.1111/jfb.12699

Nilsson PA, Huntingford FA, Armstrong JD (2004) Using the functional response to determine the nature of unequal interference among foragers. Biology Letters 271:334-337.

https://doi.org/10.1098/rsbl.2004.0170

Rands SA, Pettifor RA, Rowcliffe JM, Cowlishaw G (2006) Social foraging and dominance relationships: The effects of socially mediated interference. Behavioral Ecology and Sociobiology 60:572-581. https://doi.org/10.1007/s00265-006-0202-4

Ranta E, Nuutinen V (1985) Foraging by the Smooth Newt (Triturus vulgaris) on Zooplankton: Functional Responses and Diet Choice. Journal of Animal Ecology 54:275-293. https://doi.org/10.2307/4638 Royauté R, Berdal MA, Garrison CR, Dochtermann NA (2018) Paceless life? A meta-analysis of the pace-of-life syndrome hypothesis. Behavioral Ecology and Sociobiology 72:64. 
https://doi.org/10.1007/s00265-018-2472-z

Schröder A, Kalinkat G, Arlinghaus R (2016) Individual variation in functional response parameters is explained by body size but not by behavioural types in a poeciliid fish. Oecologia 182:1129-1140. https://doi.org/10.1007/s00442-016-3701-7

Shrader AM, Kerley GIH, Brown JS, Kotler BP (2012) Patch Use in Free-Ranging Goats: Does a Large Mammalian Herbivore Forage like Other Central Place Foragers? Ethology 118:967-974. https://doi.org/10.1111/j.1439-0310.2012.02090.x

Shrader AM, Kotler BP, Brown JS, Kerley GIH (2008) Providing water for goats in arid landscapes: effects on feeding effort with regard to time period, herd size and secondary compounds. Oikos 117:466-472. https://doi.org/10.1111/j.2007.0030-1299.16410.x Sih A, Bell A, Johnson JC (2004) Behavioral syndromes: an ecological and evolutionary overview. Trends in ecology \& evolution 19:372-378. https://doi.org/10.1016/j.tree.2004.04.009 Stears K, Kerley GIH, Shrader AM (2014) Group-Living Herbivores Weigh Up Food Availability and Dominance Status when Making Patch-Joining Decisions. PLoS ONE 9:e109011. https://doi.org/10.1371/journal.pone.0109011 Stephens DW, Krebs JR (1986) Foraging theory. Princeton University Press. Stillman RA (1996) Shape of the interference function in a foraging vertebrate. Journal of Animal Ecology 65:416-420. https://doi.org/10.1111/j.1365-2664.2005.01116.x

Toscano BJ, Griffen BD (2014) Trait-mediated functional responses: predator behavioural type mediates prey consumption. Journal of Animal Ecology 83:1469-1477. https://doi.org/10.1111/13652656.12236

Zuur AF, Ieno EN, Walker NJ, Saveliev AA, Smith GM (2009) Mixed effects models and extensions in ecology with R. Springer Verlag. 